\title{
Significance of Enhancement Technique In Segmentation of Image and Signal: A Review of the literature
}

\section{Ghada Mohammad Tahir Kasim Aldabagh ${ }^{*}$, Ashraf Abdulmunim Abdulmajeed ${ }^{2}$, Haleema Essa Solayman ${ }^{3}$}

${ }^{1 *, 2}$ Department of Software, College of Computer Sciences and Mathematics, University of Mosul, Mosul, Iraq.

${ }^{3}$ Department of Computer Sciences, College of Computer Sciences and Mathematics, University of Mosul, Mosul, Iraq.

E-mail: ${ }^{*}$ ghadaaldabagh@uomosul.edu.iq, ${ }^{2}$ ashraf althanoon@uomosul.edu.iq,

3haleema_essa@uomosul.edu.iq

(Received December 16, 2020; Accepted March 25, 2021; Available online September 01, 2021)

DOI: 10.33899/edusj.2021.129161.1134, (C) 2021, College of Education for Pure Science, University of Mosul.

This is an open access article under the CC BY 4.0 license (http://creativecommons.org/licenses/by/4.0/).

\begin{abstract}
:
From the last 70 years, there is continuous development in the field of digital image processing such as geology, biology as well as in medical fields. Solving many problems in the case of numerous application image processing plays an important role. Recently, wireless communication has been a dominant medium. When a signal or image is transmitted via the wireless environment, the quality of the image or signal gets degraded. It is the biggest issue. This happens because of acquisition and color space conversion. Hence, priority is given to enhance the quality of the image or signal. Enhancement is the process responsible to enhance the quality of the signal. In this paper, we focused on various enhancement techniques for image and signal enhancement. Furthermore, this study put down the result for various enhancement techniques for improvement in the image. Theoretically, the signal enhancement was discussed shortly.
\end{abstract}

Keyword: Digital image processing, Enhancement, Contrast, Histogram, Histogram equalization (HE), Contrast limited adaptive Histogram equalization (CLAHE).

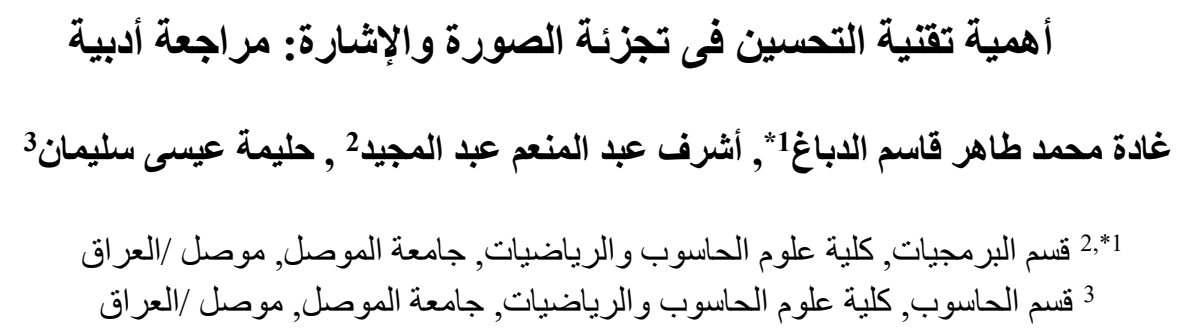

الخلاصة:

منذ 70 عامًا، كان هناك تطور مستمر في مجال معالجة الصور والاشارة الرقيمة مثل الجيولوجيا وعلم الأحياء وكذلك في الطب. حيث تلعب معالجة الصورة والاشارة الرقمية دور مهم فى حل العديد من الششاكل في صور التطبيقات ولها دور مهم. ففي الآونة

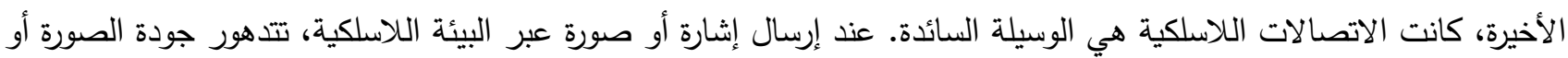

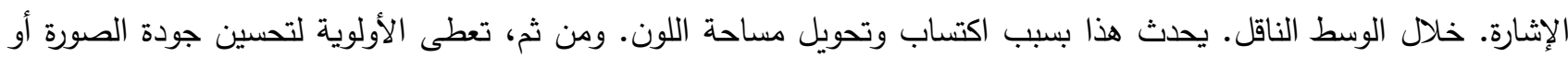




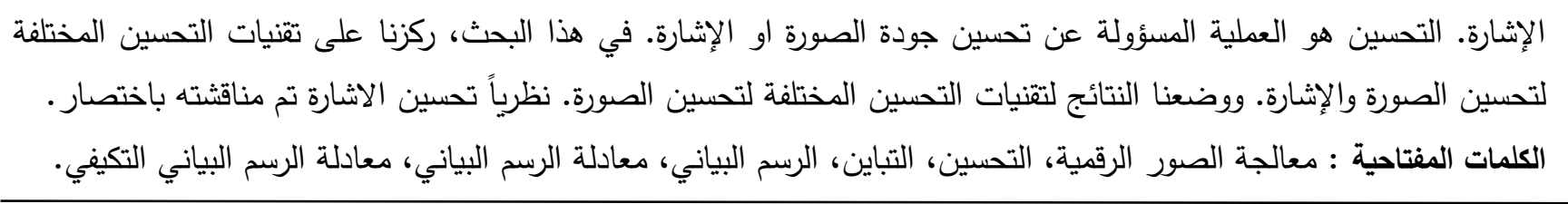

\section{Introduction:}

Image processing is an area where any experimental task is done comfortably for complex problems. It is a process where digital images are processed with various algorithms. Digital Image processing occupies different areas like remote sensing, pattern recognition, medical, video processing, and sharpening of the image. The basic flow of digital image processing is preprocessing of the image, compression of the image, Detection of edges of the image, and finally segmentation. Improvement in quality can possible by various methods. Degradation occurred in the image due to various noises and environmental effect becomes a dominant problem observe in the case of a wireless communication system. As we know the Digital image processing is the best remedy for various difficulties like poor image quality and enhancement of the degraded image. It is mainly dealing with image examination, pulls out the features (attributes), and then gives back the original image [1].

Image enhancement is a tough and important scenario in the area of digital image processing. The image enhancement technique is used for finding hidden details present in the digital image. Improvement in the quality of the image is obtained with this enhancement technique as compared to manual image presentation (representation by a human). Enhancement is the process of enhancing the features of the image. Image enhancement is generally simplification of image deals with improvement in the perception of data or information in the image for viewers. It provides better input to the image processing system. Generally, the image is captured from the natural environment with a higher dynamic range. It includes dark as well as bright regions. Human eyes have a dynamic range which makes the sensing of images difficult.

Image enhancement is a very popular method for improving the quality of an image. It is also responsible to modify the attributes of the image which is resulted in the most suitable result for the operation as well as specific spectator. Enhancement techniques sharpen the edges, boundaries, and contrast by making the image clearer and helpful for further processing or analysis. An increase in the content of data of image does not happen by enhancement technique, it only helps to increase the dynamic range of attributes which are choosing for enhancing so it becomes easy to detect. There are various ways constitutes the process of image enhancement which are responsible for the best visual appearance of the image by simple processes such as increment, in contrast, removal of noise, removal of blurred effect, and highlighting the detail [2].

Similar to image enhancement, Signal enhancement is an important scenario. In the wireless environment, the audio or other signal get degrade due to external noises, spikes, and or unwanted signals. Hence the original signal's quality gets lost. Hence it is necessary to remove that signal and enhance the quality of the received signal [3].

This paper focused on the significance of an enhancement technique. There are main two types of enhancement techniques, i.e. The spatial domain and frequency domain. The spatial domain is again classified in other types such as Histogram Equalization, Adaptive Histogram Equalization, Bihistogram Equalization, and Laplacian whereas the frequency domain technique is based on the Fourier transform [2] and [4]. Here we put the result for various enhancement techniques which shows the contrast adjustment and sharpens easily detectable edges. The theoretical part of signal enhancing is also discussed. Prominently image enhancement is discussed. The rest of the paper is organized as: the literature review is done in section Two. Section Three discusses the Basic concepts such as Basic steps of image processing, the importance of enhancement for image and signal, enhancement and its types, histogram, various enhancement techniques, signal processing, and its types. Section Four to ninth includes proposed techniques along with the result obtained by various enhancement techniques. 
Finally, the conclusion of the Reviewin Section ten,followed by acknowledgment and a list of references

\section{Literature survey:}

Many researchers were done to work on enhancement. Here discussions of some papers are carried out as follows. Ravichandran K.S. [5] et. was reported that image enhancement is responsible to enhance the visual appearance and quality of the image in 2015. They deal with improving clearance of image for human viewing, removal of blurring and noise [5]. Image enhancement is the simplest technique and is popularly applied in the digital image processing field. This statement was verified by Garg et.al in 2011[6]. Mishra and Sharma were done review various enhancement techniques along with comparisons between spatial domain and frequency domain [2]. Detailed application of enhancement of image (medical field) was discussed by Premladha et.al in 2015. They did the detection as well as the analysis of the image [7]. Nair et.al deal with Adaptive histogram equalization and verified that it gave better results in 2011 [8]. Global histogram equalization was discussed by Peli et.al. They reported that this technique adjusts intensity histogram closer to uniform distribution [9]. Dynamic histogram equalization was suggested by Chae et.al, which can control the issues of the traditional method of histogram equalization. It gave the image enhancement without losing any details contained in the image [10]. Fuzzy techniques are also used for improving the quality of the image. There are various methods (like fuzzy image segmentation, fuzzy contrast adjustments, fuzzy edge detection) are available which supports fuzzy image enhancement was suggested by Maheshwari et.al [11].

Salt and pepper type noise is removing with the help of fuzzy-based algorithms since various fuzzy filters can be designed for the removal of different types of noises. This concept was discussed by Sinha et al [12]. Bora was done the comprehensive as well as the comparative study [13]. The signal and various types of noises are discussed by the Sanford research system in [3]. Sangeetha and Kannan have reported the speech signaling with the digital filter in [14].

\section{Background concepts:}

Under this section, discussion of various points like steps of digital image processing, the importance of enhancement technique, different types of enhancements, histogram and various enhancement techniques, signal processing, signal enhancement, the importance of signal enhancement, various types of signal enhancement techniques are carried out.

\subsection{What is digital image processing}

Image processing is needed to get quality output. There are two types of image processing, analog image processing, and digital image processing. Digital image processing allows using the advanced computerized technique. They have a provision of transform images into numerical form as well as in 2D form. Quality output is obtained from the degraded raw image data is the main feature of image processing. In the form, the Finite value matrix represents an image. That matrix element is known as a pixel. A pixel is the representation of the junction point created by row and column intersection. Pixels are related to brightness value or digital number. Generally Digital image may have 6-bit data (0-63), 8-bit data (0-255) or 10-bit data (0-1024) [15]. Assume that image is conjectural (The conjectural stands for continuous, have discrete values with 2 variables), unseen (physical parameters) and insightful. The image has a unique value that falls into the category of scalar image. The image with more parameters or values falls in the category of vector image. Image is written in mathematical form as image $=p * q$. Each array element represents the data of that color pixel [16].

\subsection{Basic steps of image processing:}

The flow of image processing is digital image, enhancement, Interpretation, and reinstate image in Figure 1. 


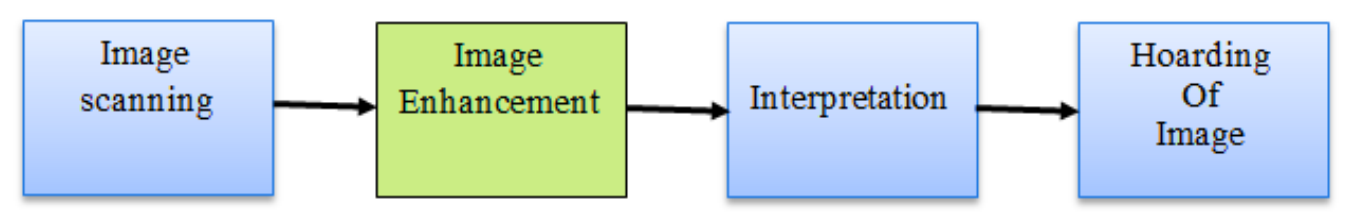

Figure 1. Basic steps in image processing

This paper deal with the second block of the above Figure 1. So first, focus on the importance of enhancement [17].

\subsection{Importance of enhancement for image and signal}

Vast aggregation of data will present in the color image. These data are hiding for security purposes hence the human eyes cannot able to identify the data or cannot analyze the image. The most significant and difficult issue is to realize minor changes or alterations in characteristics (color, intensity, texture, edges) of the given image. Quality of image plays an important role as it decides the result of an image segmentation process. Satellite image degradation occurs by a reason of noise that is mixed up during the process of capture, transmission (dissemination), and acquisition of images. Hence such noise contained image segmentation does not give an effective result of the analysis. So it is a must to have an efficient segmentation technique for color images for analysis of those changes. Segmentation of color images is an important aspect for emerging research in the field of recognition of pattern and color images. Many algorithms are available to solve this task. But the segmentation process resulted in miss classification as well as over-segmentation. There is a need to enhance the image and hence the image enhancement technique comes in the picture. The enhancement technique can remove noise or redundant pixels from the color image before the process of final segmentation. Image Enhancement becomes a very good preprocessing technique that suppresses noise with the preservation of edges as well as detailed information [13].

\section{Enhancement}

Definition: Image enhancement

It is the process to adjust the digital image in such a way that it resulted in proper form for further analysis purposes. Image enhancement is popularly useful in computer graphics. It plays as a subarea in the field of image processing. Enhancing tiny parts in the given image will depending on intensities i.e. gray level of a particular point. This will come under the concept of point processing.

Bellows Figure shows how the enhancement technique responsible to increase the quality of the image. In Figure 2. ' $a$ ' image is the original image while ' $b$ ' image represents the enhanced image. Image ' $b$ ' is a clearer image than the original image. The result obtained with the original image is worst as compared to the image processed with enhanced image ' $b$ '. 


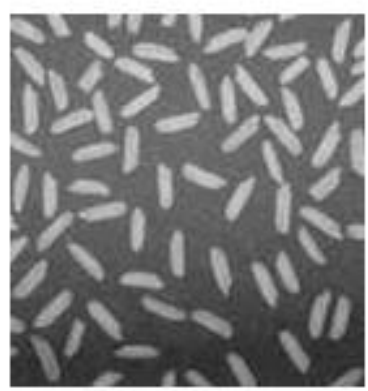

Original image

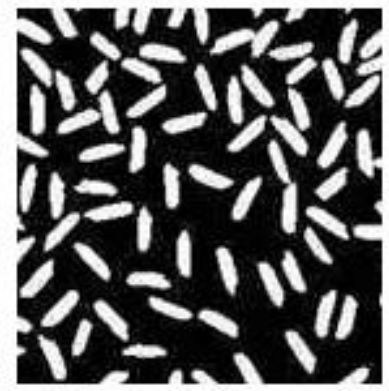

Enhanced image

Figure 2. Image enhancement

There are various types of enhancement types spatial domain, frequency domain, and color enhancement.

\section{Contrast enhancement in spatial domain:}

It is the process where the pixel intensity of the image is going to change for utilization of max possible bins [18]. The separation of bright and dark areas from the image is termed as a contrast. Removal of ambiguity which may observe in a different region of the image is the main advantage of the contrast enhancement. Figure 3 shows the original image and contrast-enhanced image. The contrast-enhanced image has nice visibility and clearness as compare to the original image.

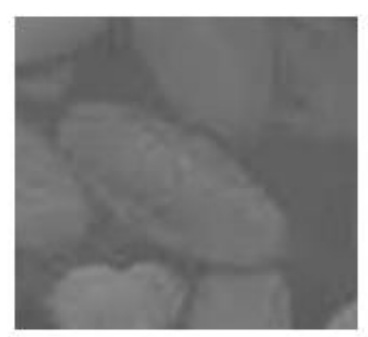

Original image

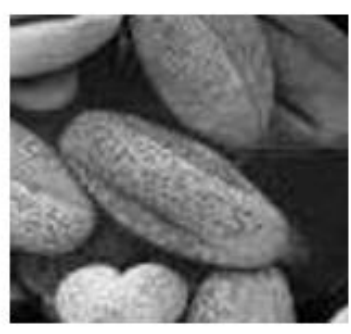

Contrast-Enhanced image

Figure 3. Contrast enhancement

Contrast enhancement is divided into two classes one is local contrast enhancement and the other is global contrast enhancement.

\subsection{Local contrast environment:}

This is an easy way of enhancement. The small window is the main part of this enhancement. A small window is sliding sequentially over each pixel of the image and enhance only those block who comes under that window. It means, there is intelligence use of local information for this case with improvement in local brightness issue for the future. Hence the contrast ratio goes on improving in each region of an image. This technique has more computational complexity. It becomes highly expensive in cases where the overlapping of the sub-blocks is obtained. Adaptive Histogram Equalization (AHE) and Contrast Limited Adaptive Histogram Equalization (CLAHE) are techniques based on local enhancement techniques which adopted frequently. 


\subsection{Global contrast environment:}

Information of global histogram is taken into the consideration for enhancement purposes. Here local information is ignored completely as a complete image is takes into consideration. This enhancement has a limiting contrast ratio for some parts of an image. It has resulted in important contrast losses from a small area of an image; particularly in the background.it is more advantageous as it has low computational complexity, simple, and suitable for the complete enhancement of the image. Histogram Specification and Histogram Equalization are the two extensively adopted under the global enhancement technique [19].

\subsubsection{Edge enhancement:}

It is a filter for image processing that is responsible to increase the contrast for any shape border in an account to improve the visible sharpness (acutance) which is resulted in the improvement in the perceived quality of the image.it can be applied to both images and video. Few cameras are available in the market which is self-adjustment in the edges of an image. It may be a digital or analog process. Bellows Figure 4 represents the changes in the edges of the circle. An image that is processed under edge enhancement technique provides more sharp edges and easily identified when viewing from a finite distance [1].

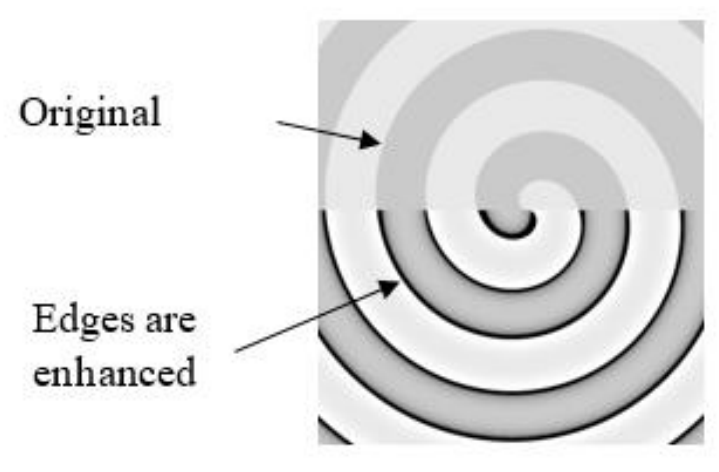

Figure 4. Edge enhancement

Here the filter is working at sharp edges boundaries mean edge between the object and background. Then it increases the contrast of the image in a region around the edges. This is nothing but the effect for the creation of subtle dark and bright highlights on both sides of the edge termed as undershoot and overshoot. Edge enhancement technique can change based on a parameter such as an Amount Radius or aperture and threshold. The Common algorithm used for of is unsharp masking.

\subsubsection{Shadow and highlight enhancement}

This enhancement technique is used for correcting exposure. It is a popular enhancement technique in the field of media, magazine cover. This technique can be implemented using adaptive histogram equalization or contrast limiting adaptive histogram equalization. As shown in Figure 5 the original image and image after adjustment in shadow and brightness. 


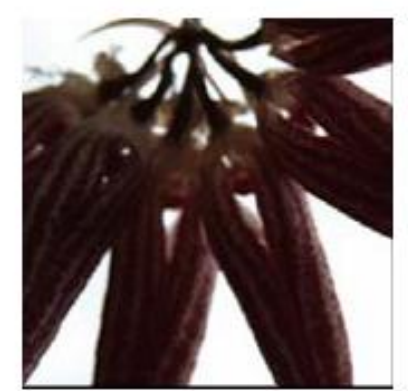

Original image

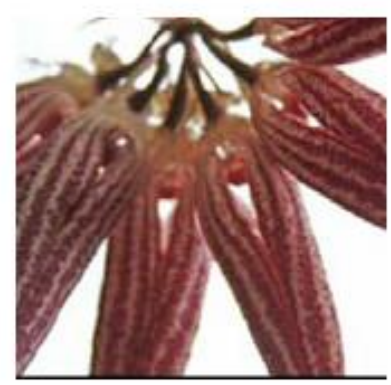

Shadow and highlighted image

Figure 5. Image enhancement

\section{Histogram:}

All the intensities present in an image are represented graphical manner as a histogram. Intensity means lightness or color distribution in the image. It is plotting the number of a pixel for every color value. The histogram represents the distribution of the gray levels present in the image and provides a global description of their appearance. Brightness or darkness, the contrast of the image can be adjusted based on histogram details so there is a clearer visual appearance of the image. Figure 6 shows the histogram [20].
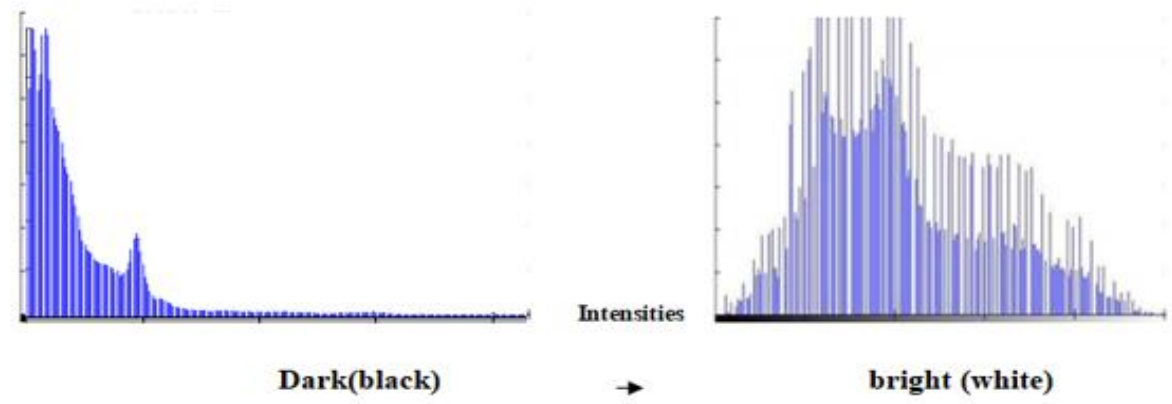

Figure 6. Histogram

\section{Various Enhancement techniques:}

Image enhancement techniques are classified into two main categories: Spatial domain techniques (it direct operates on pixels) and Frequency domain techniques (Fourier transform is the base for it) [21].

\subsection{Spatial domain techniques}

In this technique pixel values are going to manipulate. The spatial domain technique is again divided into two enhancement techniques i.e. global image enhancement and local image enhancement. Histogram equalization. adaptive histogram equalization (contrast adjustment), bi histogram equalization, and Laplacian (used for edge enhancement) are some techniques that come under spatial domain techniques.

Equation 1 represents the operation in the spatial domain

$$
g(x, y)=h(x, y) * f(x, y)
$$

where $\mathrm{g}(\mathrm{x}, \mathrm{y})$ : the resulted gray image 


$$
\begin{aligned}
& h(x, y) \text { : the original image } \\
& f(x, y) \text { : the filter }
\end{aligned}
$$

This technique is useful in alteration of values of the grey level of the separate pixel. Hence we got the complete contrast of the full image. This technique is not applicable for an effective method as it cannot selectively enhance the edges or for the required part of information [8]

\subsection{Frequency domain techniques}

Frequency domain techniques are operating on the Fourier transform of the image. Image enhancement in this domain was observed straight forward. Initially, the image is converted in the frequency domain. In this technique there is the simple computation of Fourier transform to enhance, then multiply it with filter and finally with help of inverse transform produced enhanced image i.e. resultant image.

This method is preferably used for modification of brightness and contrast of the image. As a sequel, output pixel value I going to modify as per transformation function which is applied to input image values [22]. Mathematical frequency domain relation is represented by equation 2 which is the convolution theorem

$$
\mathrm{G}(\mathrm{u}, \mathrm{v})=\mathrm{H}(\mathrm{u}, \mathrm{v}) \mathrm{F}(\mathrm{u}, \mathrm{v}) \ldots \ldots(2)
$$

Here $\mathrm{G}, \mathrm{H}$, and $\mathrm{F}$ are Fourier transform of $\mathrm{g}$, h, and f respectively.

This technique is useful in the enhancement of edges as well as selective information. The entire image cannot enhance in this technique.

Here a few enhancement techniques are listed which are highly preferable. Filtering with morphological operators, Histogram equalization, Wiener filter (used for Noise removal), linear contrast adjustment, Median filtering, Unsharp mask filtering, adaptive histogram equalization, Contrast-limited adaptive histogram equalization (CLAHE), and Decorrelation stretch.

\section{What is signal processing?}

Signal processing encompasses the artificial signal in a useful form for a particular application purpose. The signal may be audio, speech, video, or any other. Speech processing study includes compression, modeling, and recognition. Signal processing is applied to analog signals. It operates on $1 \mathrm{D}$ or $2 \mathrm{D}$ signals. The image is manipulated or converted into electrical signals (sine and cosine waves) [3] and [23].

\subsection{Signal enhancement and the importance of signal enhancement.}

Any audio, video, sensor output, or image is converted into an appropriate electrical signal then it is processed. When the signal is getting from the natural environment it is in impure form as it contains some noises which are due to the wireless environment. To remove unwanted noise, spikes from the input for further processing to get a quality signal. 


\subsection{Various types of signal enhancement techniques}

\subsubsection{Acoustic enhancement:}

It is also known as posterior enhancement (enhance through transmission) mention for increasing echoes deeper to structures that transmit sound unusually well. Used to analyze the characteristic of the urinary bladder, cysts, and gallbladder.

\subsubsection{Blip enhancement:}

It is useful in security systems. The electronic warfare method is used for making fool radar. In that case, blip enhancement is used.

\subsubsection{Speech enhancement:}

It is also an important concept in increasing speech quality in presence of audio signal processing.

\subsubsection{Forensic audio enhancement:}

For scientific analysis as well as improving audio clarity is the critical analysis particularly in case of improvement in intelligibility. Such cases can be analyzed with Forensic audio enhancement techniques.

\subsubsection{Orchestral enhancement:}

Modification in the live performance of music can be obtained with help of Orchestral enhancement. Here electronic sound augmentation is used for modification purposes [23].

\section{Proposed techniques:}

In the previous section, the discussion is done on many types of image and signal processing techniques. Here proposed techniques used to study the enhancement techniques are contrast limited adaptive histogram equalization, and Histogram equalization.

\subsection{Contrast Limited Adaptive Histogram equalization:}

Is technique falls under contrast enhancement? In this method, the image is divided into many sub-images (That sub-images are known as tiles) and the $\mathrm{n}$ histogram computation is done to get cumulative distribution function (CDF). Clipping of histogram is obtained for predefined values for limiting the amplification. The swarm generated because of the clipped bins is dispensed over the histogram of that tile. Image enhanced by CLAHE is good as compared to image enhanced by histogram equalization. Some gray level transformations are used in contrast enhancement shown in Figure 7. 


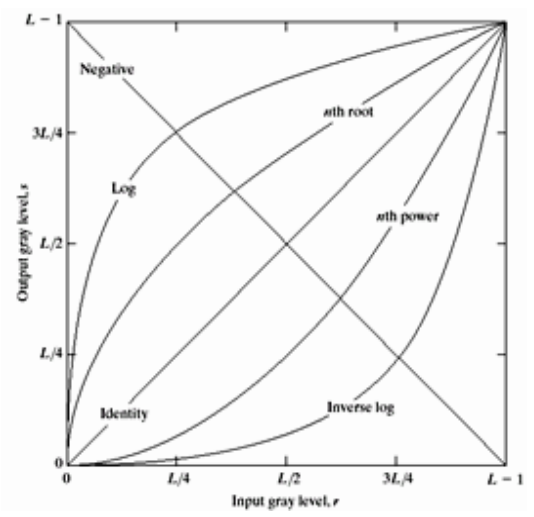

Figure 7. Basic grey level transformation

\subsection{Histogram equalization}

Histogram equalization is a popular and easy technique used in contrast enhancement. It provides a basic overview of the image which is enhanced. HE is going to enhance the contrast of the image by flatting density distribution for the resultant image. HE supports the stretching of the dynamic range. The stretching concept is shown in Below Figure 8.
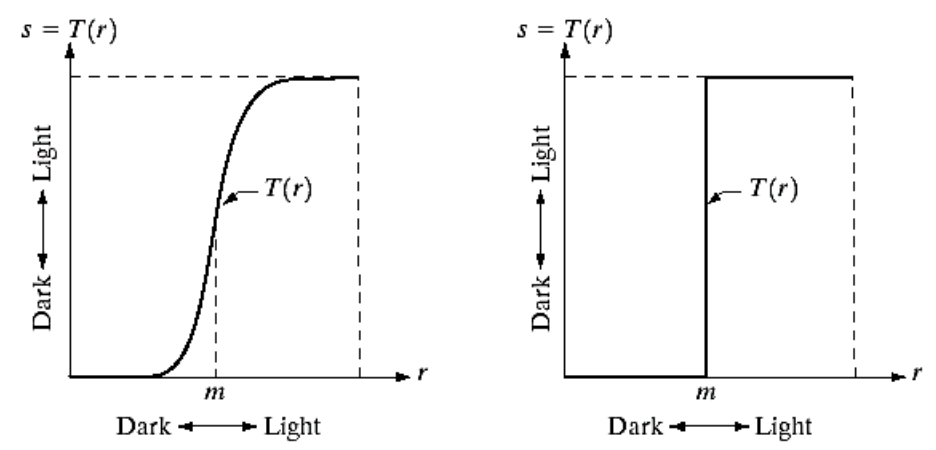

Figure 8. contrast stretching

Wheres $=$ Output image pixel value and $r=$ input image pixel value

High performance in the case of contrast enhancement of an image is observed in HE. In this method alteration of original brightness of input image is done, worsened visual quality with introducing few undesirable artifacts. So this method is not much preferable or suitable in some images where original details and brightness are essential. This technique is broadly classified into two types as global histogram equalization and local histogram equalization [24] and [25].

The non-linear transformation $g=T(f)$ is applied to every pixel of input image $f(x, y)$ for uniform grey level distribution which is resulted in $\mathrm{g}[\mathrm{x}, \mathrm{y}]$.

For both these techniques. The program is done in MATLAB to observe the effect of enhancement on the input image. 
Journal of Education and Science (ISSN 1812-125X), Vol: 30, No: 4, 2021 (15-27)

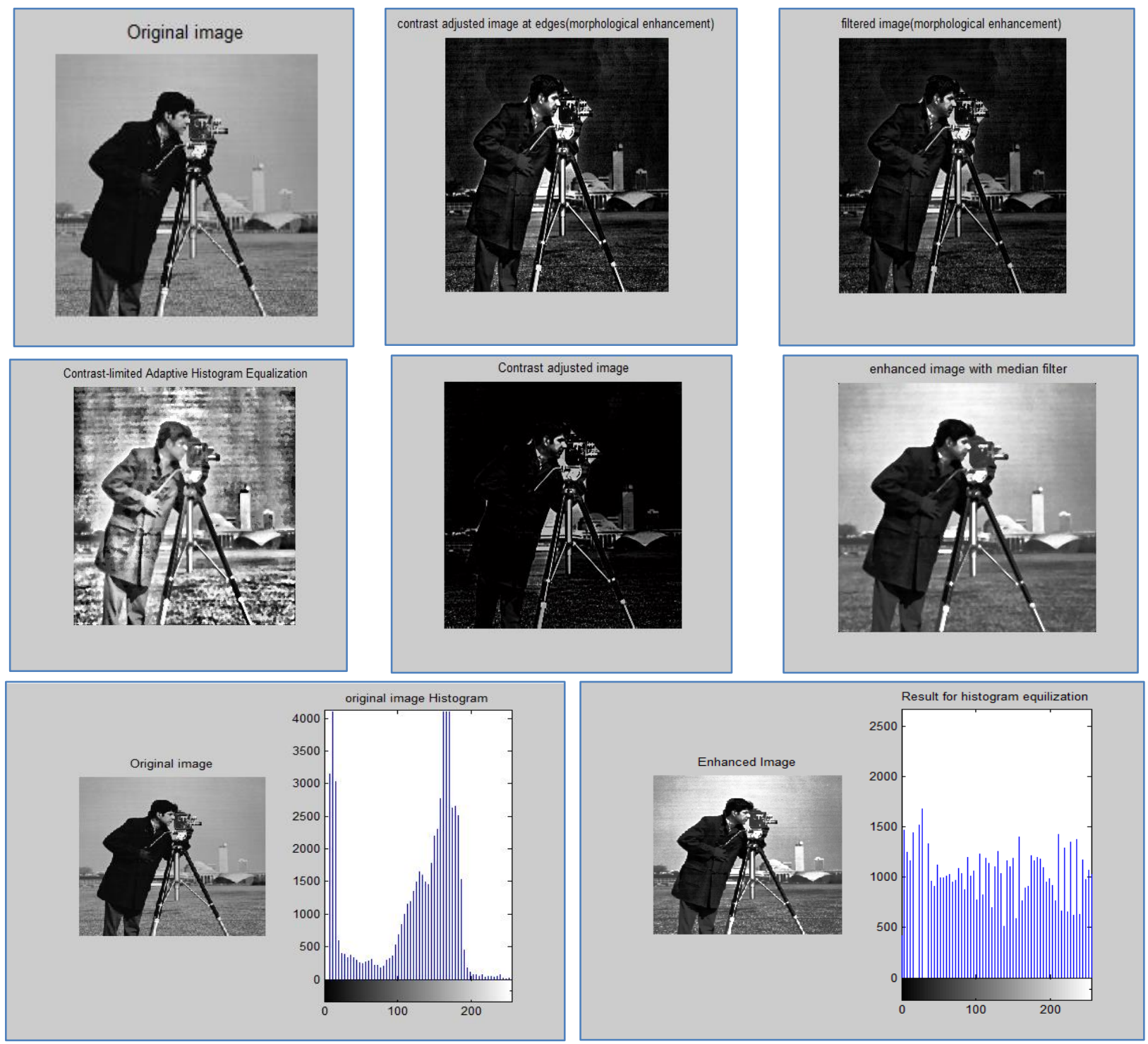

Figure 9. Result for Contrast Enhancement Techniques

\section{Conclusion}

Image enhancement is playing important role in the field of image processing. This paper has a deal a review of the various types of enhancement techniques. Few techniques of image processing have been done which are not giving proper results. Most of the techniques are deal with the overall contrast adjustment of the image as they do the alter of grey levels value for every pixel. Depending on the application field and comparison between the various techniques. we can select the appropriate enhancement technique. Here we just reviewed and study the theoretical part of signal processing. Also studied some signal enhancement techniques shortly.

\section{Acknowledgments}

The authors would like to thank the University of Mosul / College of Computer Science and Mathematics for their facilities, which have helped to enhance the quality of this work. 


\section{References}

[1] S. Muthuselvi and P. Prabhu,"Digital Image Processing Techniques - A Survey", International Multidisciplinary Research Journal, 5(11), pp.1-11, (2016).

[2] Ruchika Mishra and Utkarsh Sharma, "Review of Image Enhancement Techniques". International Journal of Engineering Research \& Technology (IJERT), 2(8),pp.846-851, (2013).

[3] Shyang-Jye Chang and Hung-Wei Hsieh, "Development of the Audio Enhancement Method by Using the Reflected Signals in the Reverberant Environment", Hindawi Publishing Corporation, Article ID 385196, 6 page, (2015).

[4] Ravinder Kaur and Taqdir," Image Enhancement Techniques- A Review" .International Research Journal of Engineering and Technology (IRJET), 3(3), pp.1308-1315, (2016).

[5] K.S.Ravichandran, P.Janani, J.Premaladha, "image enhancement techniques: A Study", Indian Journal of Science and Technology, Vol 8(22) September, (2015).

[6] Rajesh Garg, Bhawna Mittal, Sheetal Garg, "Histogram Equalization Techniques for Image Enhancement". IJECT, Vol.2 Issue 1, ISSN 2230-9543, (2011).

[7] W. Rui, and W. Guoyu, "Medical X-ray image enhancement method based on TV homomorphic filter". IEEE, International Conference on Image, Vision and Computing (ICIVC), 2nd Int. Conf. on. pp. 315-318 (2017).

[8] Arun R, Madhu S.Nair,R.Vrinthavani and Rao Tatavarti, "Alpha Rooting Based Hybrid Technique for Image Enhancement". Online publication in IAENG, (2011).

[9] Jinshan Tang Eli Peli and Scott Acton, "Image Enhancement Using a Contrast Measure in the Compressed Domain" ,IEEE Signal processing Letters, Vol.10, No. (2003).

[10] M.Abdullah Al-Wadud, Md.Hasanul Kabir, M.Ali Akber Dewan, and Oskam Chae,"A Dynamic Histogram equalization for image contrast enhancement", IEEE Trans.Consumer Electron,Vol.53, no.2, pp 593-600, (2007).

[11] Tarun Maheshwari, Amit Asthana, "Image ehnacement using fuzzy techniques". IJRREST, Vol.2, Issue-2, (2013).

[12] G. R. Sinha and N. Agrawal, "Fuzzy based Image Enhancement Method", IJCA, pp. 13-18, (2015).

[13] DIBYA JYOTI BORA,"Importance Of Image Enhancement Techniques In Color Image Segmentation: A Comprehensive And Comparative Study". Indian Journal of Scientific Research, pp.1-27, (2017).

[14] S. Sangeetha and P. Kannan, "Design And Analysis Of Digital Filters For Speech Signals Using Multirate Signal Processing". ICTACT Journal On Microelectronics,3(4), pp.480-487, (2018).

[15] Muzamil Bhat, "Digital Image Processing". International Journal Of Scientific \& Technology Research, 3(1), pp.272-276, (2014).

[16] Alexandrina-Elena P., Mihai B. And Gabriela C, "Image Processing Using Artificial Neural Networks", Bul. Inst. Polit. Iaşi, t. LXI (LXV) pp.9-21, (2015).

[17] Ravindra S. Hegadi, "Image Processing: Research Opportunities and Challenges". National Seminar on Research in Computers, Bharathiar University, Coimbatore, India 3, (2010).

[18] Gupta, S., \& Kaur, Y, "Review of Different Local and Global Contrast Enhancement Techniques for a Digital Image". International Journal Of Computer Applications, 100(18), pp.18-23, (2014).

[19] Aditi Majumder, "Contrast Enhancement of Images using Human Contrast Sensitivity". Computer Science Department, University of California, Irvine Sandy Irani, pp 1-9, (2006).

[20] Harpreet Kaurb and Neelofar Sohi, "A Study for Applications of Histogram in Image Enhancement", The International Journal of Engineering and Science (IJES), 6(6),pp59-63, (2017). 
[21] Ravinder Kaur and Taqdir, "Image Enhancement Techniques- A Review". International Research Journal of Engineering and Technology (IRJET), 3(3), pp.1308-1315, (2016).

[22] Komal R. Hole, Vijay S. Gulhane, Nitin D. Shellokar, "Application of Genetic Algorithm for Image Enhancement and Segmentation", IJARCET, vol.2, issue. 4, (2013).

[23] Koen Eneman Et al, "Evaluation Of Signal Enhancement Algorithms For Hearing Instruments". Research gate, pp1-5, (2014).

[24] Joonwhoan Lee, Suresh Raj Pant, and Hee-Sin Lee, "An Adaptive Histogram Equalization BasedLocal Technique for Contrast Preserving Image Enhancement". International Journal of Fuzzy Logic and Intelligent Systems, 15(1), pp. 35-44, (2015).

[25] Ravindra Pal Singh and Manish Dixit,"Histogram Equalization: A Strong Technique for Image Enhancement". International Journal of Signal Processing, Image Processing and Pattern Recognition, Vol.8, No.8, pp.345-352, (2015). 